Case Report

\title{
Novel Pathogenic Variant in TGFBR2 Confirmed by Molecular Modeling Is a Rare Cause of Loeys-Dietz Syndrome
}

\author{
Michael T. Zimmermann, ${ }^{1}$ Raul A. Urrutia, ${ }^{2}$ Patrick R. Blackburn, ${ }^{3}$ Margot A. Cousin, ${ }^{4}$ \\ Nicole J. Boczek, ${ }^{4}$ Eric W. Klee, ${ }^{1,4}$ Colleen Macmurdo, ${ }^{5}$ and Paldeep S. Atwal ${ }^{3,5}$ \\ ${ }^{1}$ Division of Biomedical Statistics and Informatics, Department of Health Sciences Research, Mayo Clinic, Rochester, MN, USA \\ ${ }^{2}$ Laboratory of Epigenetics and Chromatin Dynamics, Departments of Biochemistry and Molecular Biology, Biophysics, and Medicine, \\ Mayo Clinic, Rochester, MN, USA \\ ${ }^{3}$ Department of Clinical Genomics, Center for Individualized Medicine, Mayo Clinic, Jacksonville, FL, USA \\ ${ }^{4}$ Center for Individualized Medicine, Department of Clinical Genomics, Mayo Clinic, Rochester, MN, USA \\ ${ }^{5}$ Division of Medical Genetics, Department of Pediatrics, Stanford University, Stanford, CA, USA
}

Correspondence should be addressed to Paldeep S. Atwal; paldeep.atwal@gmail.com

Received 26 October 2016; Accepted 22 December 2016; Published 9 January 2017

Academic Editor: Philip D. Cotter

Copyright (c) 2017 Michael T. Zimmermann et al. This is an open access article distributed under the Creative Commons Attribution License, which permits unrestricted use, distribution, and reproduction in any medium, provided the original work is properly cited.

\begin{abstract}
Loeys-Dietz syndrome (LDS) is a connective tissue disorder characterized by vascular findings of aneurysm and/or dissection of cerebral, thoracic, or abdominal arteries and skeletal findings. We report a case of a novel pathogenic variant in TGFBR2 and phenotype consistent with classic LDS. The proband was a 10 -year-old presenting to the genetics clinic with an enlarged aortic root (Z-scores 5-6), pectus excavatum, and congenital contractures of the right 2 nd and 3rd digit. Molecular testing of TGFBR2 was sent to a commercial laboratory and demonstrated a novel, likely pathogenic, variant in exon 4, c.1061T>C, p.(L354P). Molecular modeling reveals alteration of local protein structure as a result of this pathogenic variant. This pathogenic variant has not been previously reported in LDS and thus expands the pathogenic variant spectrum of this condition.
\end{abstract}

\section{Background}

Loeys-Dietz syndrome (LDS) is a connective tissue disorder characterized by vascular findings of aneurysm and/or dissection of cerebral, thoracic, or abdominal arteries and skeletal findings comprising pectus abnormality, scoliosis, arachnodactyly, hypermobile joints, congenital contractures of digits, and talipes equinovarus $[1,2]$. In addition, craniofacial findings including hypertelorism, bifid uvula, cleft palate, and craniosynostosis are commonly observed in patients affected by LDS. Intellect is generally normal and typically patients develop arterial aneurysms with death resulting from a ruptured arterial aneurysm occurring in the third decade of life if left undiagnosed [2,3]. TGFBR1 and TGFBR2 pathogenic variants were initially shown to cause LDS, with subsequent pathogenic variants identified in SMAD3, $T G F B 2$, and TGFB3. We report a case of a novel pathogenic variant in TGFBR2 and phenotype consistent with LDS [4] and demonstrate pathogenic effects on the downstream protein structure based on novel molecular modeling techniques.

\section{Presentation}

The proband was a 10 -year-old presenting to the genetics clinic with an enlarged aortic root ( $Z$-score 5-6), pectus excavatum, and congenital contractures of the right 2 nd and 3rd digit. His pediatrician originally referred him to a local cardiologist for a suspected connective tissue disorder. $\mathrm{He}$ was born at term to a 19-year-old G2P2 mother and 31-yearold father. Birth weight and length were normal; however, he was noted to have contractures of the right $2 \mathrm{nd}$ and $3 \mathrm{rd}$ digit. Other medical history includes a mild restrictive lung disease that was felt to be secondary to his moderately severe pectus excavatum. His development had been normal and 
he was in mainstream education. A three-generation family pedigree did not reveal any consanguinity. Both parents were unaffected. Physical examination demonstrated head circumference of $50 \mathrm{~cm}$ (50th percentile), height of $150 \mathrm{~cm}$ (84th percentile), weight of $33.2 \mathrm{~kg}$ (42nd percentile), arm span of $156 \mathrm{~cm}$, arm span/height ratio of 1.04, and upper to lower segment ratio of 0.923 . He was normocephalic and hyperteloric (inner canthal distance $4 \mathrm{~cm}$, $>97$ th percentile; outer canthal distance $10 \mathrm{~cm},>97$ th percentile; interpupillary distance $6.5 \mathrm{~cm},>97$ th percentile). He had nasal asymmetry and a bifid uvula. There were marked pectus excavatum, mild scoliosis, shoulder hyperextensibility, negative wrist and thumb sign, no arachnodactyly, pes planus, and thin translucent skin over anterior chest and arms with evidence of easy bruising. Echocardiogram demonstrated normal valves with a severely dilated aortic root between 5-6 Z-scores. He was prescribed losartan $1 \mathrm{mg} / \mathrm{kg} /$ day managed by a cardiologist.

Molecular testing of a gene panel including TGFBR1, TGFBR2, SMAD3, TGFB2, and TGFB3 was performed using standard techniques via CLIA/CAP certified commercial laboratory and demonstrated a novel likely pathogenic variant in exon 4 of TGFBR2, denoted by c.1061T>C, p.(L354P). The variant is in a highly conserved region of DNA (13/13 species). The change occurs in the serine-threonine kinase domain of the protein. Parental testing performed at the same laboratory revealed this was a de novo change.

Additional findings include pulmonary function tests confirming a restrictive lung defect, likely related to the pectus deformity, not reversible by bronchodilators, for which he is followed up by pulmonology. An ophthalmological exam did not find any evidence of myopia or subluxation. An MRI/MRA head to pelvis was performed which demonstrated dolichoectasia of the internal carotid and left vertebral arteries, tortuosity of vessels within the chest, particularly vessels arising from the aortic arch, high-grade stenosis at the origins of the right common carotid and right subclavian arteries and proximal left common carotid artery, marked pectus deformity (Haller index of 13.4) causing compression of the right atrium and leftward shift of heart, and lastly a mildly tortuous left renal artery. The clinical features found in our patient were consistent with the diagnosis of LDS.

Interestingly, the patient was followed up by the allergy clinic for a history of idiopathic urticaria. The patient was placed on cetirizine and his symptoms resolved. TGFBR pathogenic variants have been shown to increase the predisposition for allergic disease (including asthma, eczema, and allergic rhinitis) in patients with LDS because of their role in directing immune responses to mucosal antigens [5]. A 2013 study suggested that treatment with losartan could modify allergic disease in patients by reducing excessive TGFB signaling in lymphocytes [5].

\section{Molecular Modeling}

We developed a homology-based model for the TGFBR2 kinase domain based on the experimentally solved structure of Activin Receptor IIA (see Figure 1) using available structural information for other members of this family of serine/threonine kinases. Ramachandran plots identified $96.5 \%$ of the residues in the allowable regions, reflecting the high quality of our model. Subsequently, we developed a model for the L354P variant by in silico site directed mutagenesis. These models were energy minimized, were heated to room temperature, and underwent short molecular dynamics simulation.

Molecular dynamics (MD) simulations were performed for our WT and L354P models using the CHARMm all-atom force-field [6] and a $2 \mathrm{fs}$ time step. A distance-dependent implicit environment model, as implemented in Discovery Studio [7], was used with a dielectric constant of 80 and a $\mathrm{pH}$ of 7.4. Each model was energy minimized for 5000 steps using steepest decent followed by 5000 steps of conjugate gradient and the SHAKE [8] procedure. Each system was independently heated to $300 \mathrm{~K}$ and equilibrated, followed by generation of $10 \mathrm{~ns}$ production simulations. Analyses were performed in the $\mathrm{R}$ programming language [9], leveraging the bio3d [10] package version 2.2.4. Molecular visualizations were generated using Discovery Studio and PyMol [11].

These simulations revealed loss of local secondary structure around L354P as well as resulting shifts in molecular flexibility. Schematic representation of the protein molecular model is shown in Figure 1. The L354P variant introduces a kink within helix E, a secondary structural feature located underneath the floor of the ATP binding site. We posit that the L354P pathogenic variant may change the dynamics or molecular flexibility of the protein, thereby impacting other key functional regions within the protein.

\section{Discussion}

LDS is a childhood onset connective tissue disorder characterized by distinctive vascular, anomalies, and molecular abnormalities. Pathogenic variants in all 5 genes that cause LDS, TGFBR1, TGFBR2, TGFB2, TGFB3, and SMAD3 result in loss of wild-type function of the protein product. The novel pathogenic variant identified in our patient is located in the intracellular serine-threonine kinase domain, which is where the majority of pathogenic variants are found. According to published data, most pathogenic variants in TGFBR2 are missense, and only a handful of nonsense pathogenic variants have been reported. It has been reported previously that the phenotype in LDS results due to excess activation and signaling by the TGF- $\beta$ growth factor $[3,12]$. Molecular confirmation is highly warranted as it can confirm a diagnosis suspected on clinical grounds and additionally it is important to identify the underlying molecular mutation in cases such as ours as it can be useful to (1) inform management, (2) confirm the diagnosis, and (3) provide actionable information for family planning purposes. Additionally, we demonstrate a novel molecular modeling technique which can be useful for determining pathogenicity of newly identified variants. This technique is applicable to many different genes and proteins and not specific to TGFBR2. In summary, we describe a patient with LDS and a novel TGFBR2 pathogenic variant, previously undescribed as causal for LDS, and thus expand the mutation spectrum of this condition. 


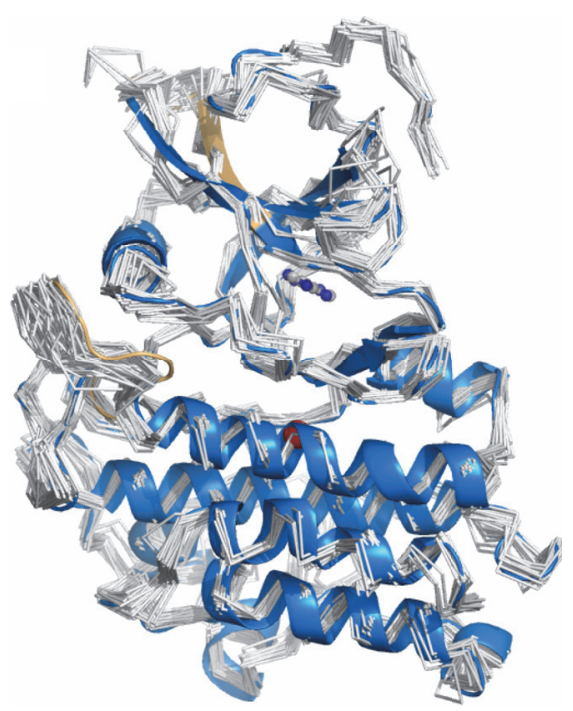

(a)

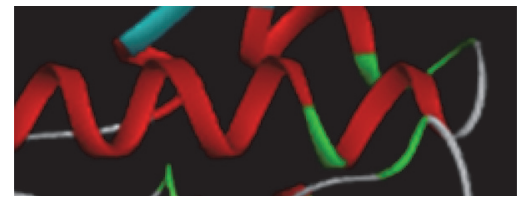

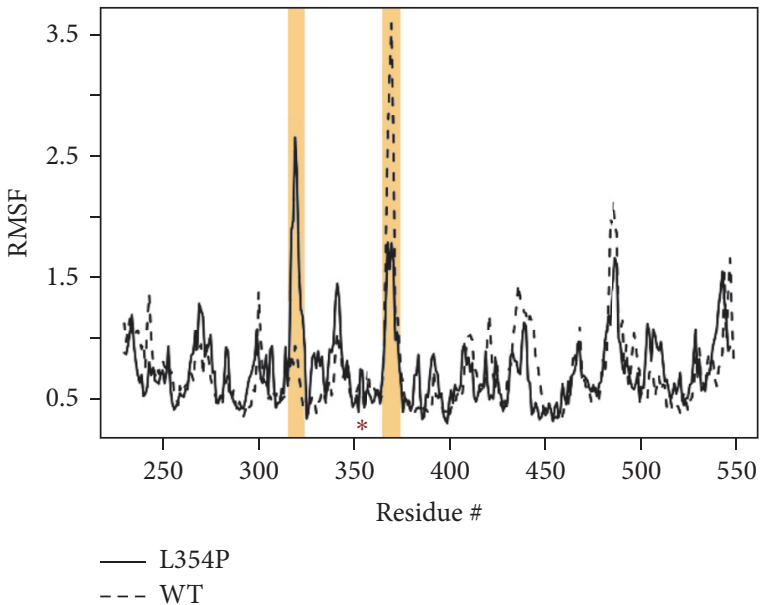

(b)

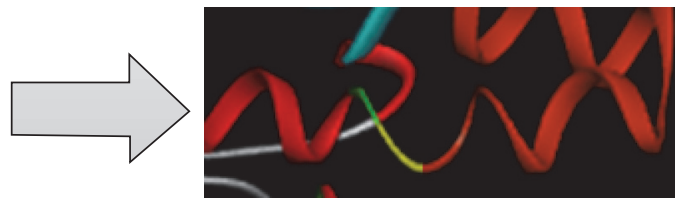

(c)

FIGURE 1: Molecular modeling of the L354P mutation reveals alteration of local structure leading to changes in flexibility. (a) The TGFBR2 kinase domain structure is shown in a blue cartoon representation with amino acid position 354 marked by a red sphere and an adenosine placed in the ATP binding pocket shown as ball-and-stick. Representatives from the beginning of our MD simulation are shown as white backbone trace. (b) Root Mean Squared Fluctuations (RMSF) capture the flexibility of the protein and are plotted for the mutant and wt simulations. Regions with largest difference are highlighted (colored similarly in (a)) and position 354 is marked with an asterisk. (c) During our simulation, the local helical structure around L354P is destabilized and unwinds, pushing the rest of the helix into an altered conformation.

\section{Abbreviations}

LDS: Loeys-Dietz syndrome.

\section{Ethical Approval}

All procedures followed were in accordance with the ethical standards of the responsible committee on human experimentation (institutional and national) and with the Helsinki Declaration of 1975, as revised in 2000 (5).

\section{Consent}

Informed consent was obtained from all patients for being included in the study.

\section{Competing Interests}

All authors declare that there is no conflict of interests.

\section{Authors' Contributions}

All authors above made substantial contributions to the conception or design of the work, or the acquisition, analysis, or interpretation of data for the work, drafting the work, or revising it critically for important intellectual content, gave final approval of the version to be published, and agreed to be accountable for all aspects of the work in ensuring that questions related to the accuracy or integrity of any part of the work are appropriately investigated and resolved.

\section{Acknowledgments}

Funding was provided by the Center for Individualized Medicine, Mayo Clinic, that provided funds to conduct the molecular modeling.

\section{References}

[1] E. Arbustini, N. Marziliano, and L. Magrassi, "Aneurysm syndromes and TGF- $\beta$ receptor mutations," New England Journal of Medicine, vol. 355, no. 20, article 2155, 2006.

[2] A. Jamsheer, C. Henggeler, J. Wierzba et al., "A new sporadic case of early-onset Loeys-Dietz syndrome due to the recurrent mutation p.R528C in the TGFBR2 gene substantiates interindividual clinical variability," Journal of Applied Genetics, vol. 50, no. 4, pp. 405-410, 2009.

[3] B. L. Loeys and H. C. Dietz, "Loeys-Dietz syndrome," in GeneReviews $^{\circledR}$, R. A. Pagon, M. P. Adam, H. H. Ardinger et al., Eds., University of Washington, Seattle, Wash, USA, 1993-2016. 
[4] M. Arslan-Kirchner, J. T. Epplen, L. Faivre et al., "Clinical utility gene card for: Loeys-Dietz syndrome (TGFBR1/2) and related phenotypes," European Journal of Human Genetics, vol. 19, no. 10,2011

[5] P. A. Frischmeyer-Guerrerio, A. L. Guerrerio, G. Oswald et al., "TGF $\beta$ receptor mutations impose a strong predisposition for human allergic disease," Science Translational Medicine, vol. 5, no. 195, Article ID 195ra94, 2013.

[6] W. D. Cornell, P. Cieplak, C. I. Bayly et al., "A second generation force field for the simulation of proteins, nucleic acids, and organic molecules," Journal of the American Chemical Society, vol. 117, no. 19, pp. 5179-5197, 1995.

[7] BIOVIA, Dassault Systèmes BIOVIA, Discovery Studio Modeling Environment, Release 2017, Dassault Systèmes, San Diego, Calif, USA, 2017.

[8] J.-P. Ryckaert, G. Ciccotti, and H. J. C. Berendsen, "Numerical integration of the cartesian equations of motion of a system with constraints: molecular dynamics of n-alkanes," Journal of Computational Physics, vol. 23, no. 3, pp. 327-341, 1977.

[9] R. C. Team, "R: A Language and Environment for Statistical Computing," 2014, http://www.R-project.org.

[10] B. J. Grant, A. P. C. Rodrigues, K. M. ElSawy, J. A. McCammon, and L. S. D. Caves, "Bio3d: an R package for the comparative analysis of protein structures," Bioinformatics, vol. 22, no. 21, pp. 2695-2696, 2006.

[11] The PyMOL Molecular Graphics System, Version 1.5.0.3. Schrödinger, LLC, https://www.pymol.org/citing.

[12] B. L. Loeys, J. Chen, E. R. Neptune et al., "A syndrome of altered cardiovascular, craniofacial, neurocognitive and skeletal development caused by mutations in TGFBR1 or TGFBR2," Nature Genetics, vol. 37, no. 3, pp. 275-281, 2005. 


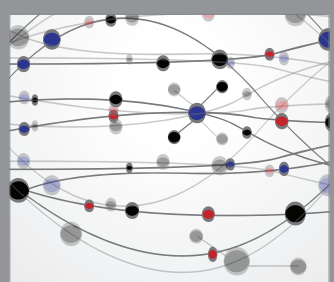

The Scientific World Journal
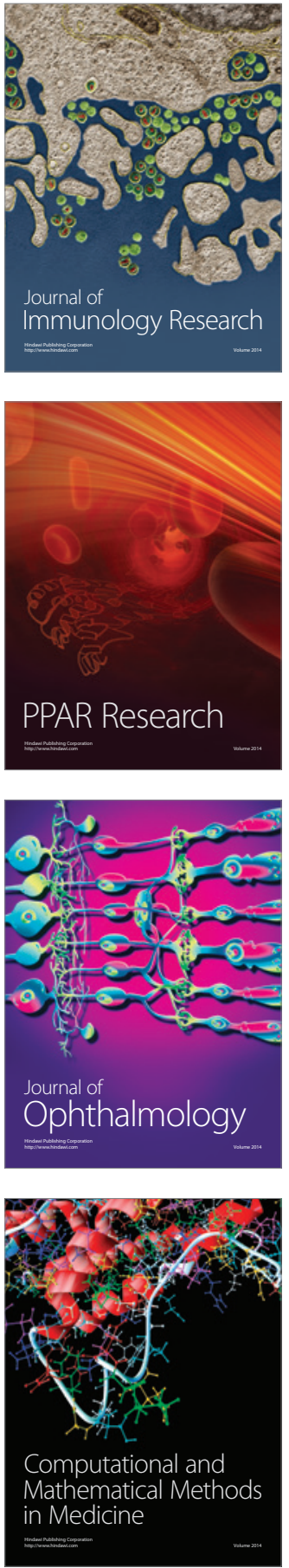

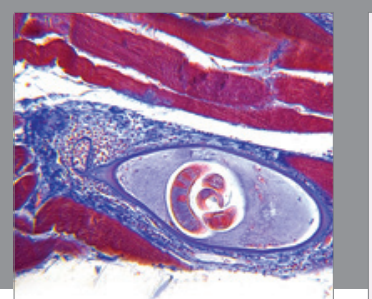

Gastroenterology Research and Practice
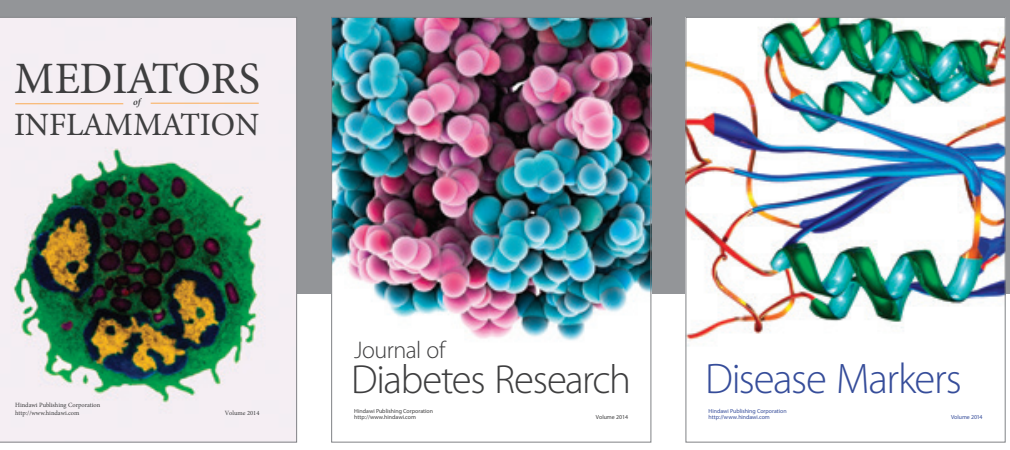

Disease Markers

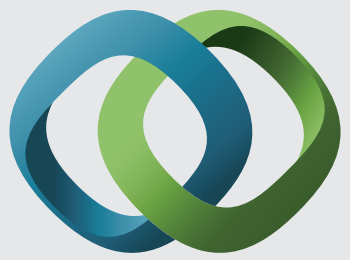

\section{Hindawi}

Submit your manuscripts at

https://www.hindawi.com
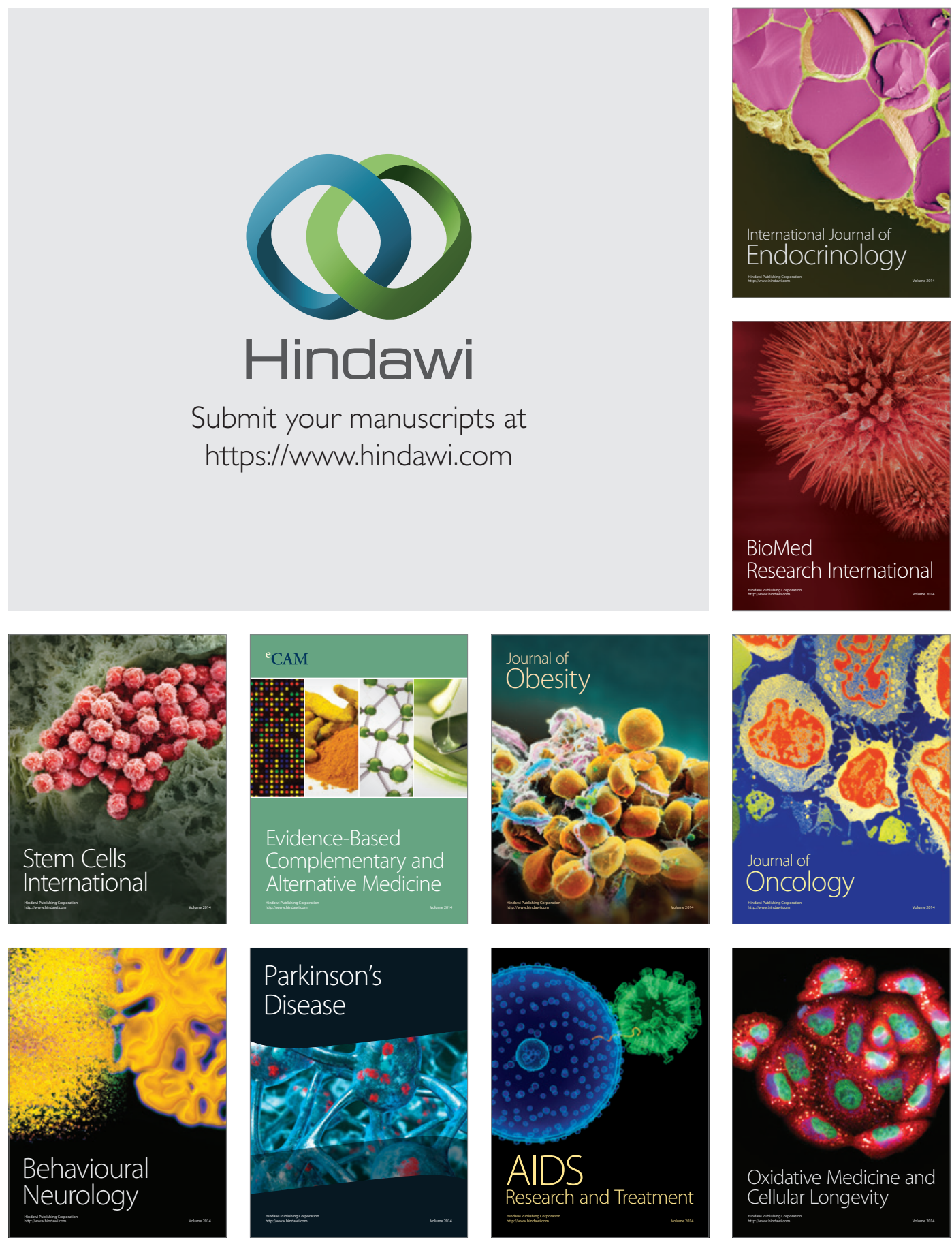